\title{
Psychedelic treatment of functional neurological disorder: a systematic review
}

\author{
Matthew Butler (iD), Mathieu Seynaeve, Timothy R. Nicholson, Susannah Pick, \\ Richard A. Kanaan, Andrew Lees, Allan H. Young (iD) and James Rucker ${ }^{(D)}$
}

\begin{abstract}
Functional neurological disorder (FND), formerly known as conversion disorder, causes a high burden of disability and distress, and is amongst the most commonly encountered conditions in neurology clinics and neuropsychiatric services, yet the therapeutic evidence base is somewhat limited. There has been recent interest in the therapeutic potential of psychedelics such as psilocybin and lysergic acid diethylamide (LSD), and in recent studies psychedelics have shown promise in treating a range of neuropsychiatric conditions. Modification of neural circuits associated with self-representation is thought to underlie some of this effect, and as some contemporary theories of FND focus on aberrant somatic self-representation, psychedelics may therefore represent an unexplored treatment option for FND. We systematically reviewed studies involving the use of psychedelics in FND. Nine studies published between 1954 and 1967, with a total of 26 patients, were identified. Due to restriction of licencing of psychedelic drugs since this period, no modern studies were identified. In most cases, patients received a course of psychotherapy with variable adjunctive administration of psychedelics (in a combination known as 'psycholytic therapy'), with protocols varying between studies. Of those treated, $69 \%(n=18)$ were found to have made at least some recovery on heterogeneous and subjective clinician-rated criteria. Adverse events were mostly mild and transient; however, at least one patient terminated the study due to distressing effects. All included studies were of low quality, often lacking control groups and valid outcome measures. Although no conclusions on efficacy may be drawn from these data, further research may help to determine whether psychedelics offer a feasible, safe and effective treatment for FND.
\end{abstract}

Keywords: psychedelics, psilocybin, LSD, functional neurological disorder, conversion disorder

Received: 23 September 2019; revised manuscript accepted: 11 February 2020.

\section{Introduction}

Functional neurological disorder (FND) constitutes symptoms of the voluntary motor or sensory nervous system that can be positively identified as being incompatible with recognised neurological diseases. FND symptoms mimic almost any neurological presentation; however, seizures, movement disorders, weakness, and sensory symptoms predominate. ${ }^{1} \mathrm{FND}$ has an estimated prevalence of 50 per 100,000 population in the community. ${ }^{2,3}$ It is one of the most common causes of referral to neurological clinics, ${ }^{3}$ a frequent cause of neurological disability, ${ }^{4}$ and has a high incidence of psychiatric comorbidity. ${ }^{5}$
Nevertheless, successful evidence-based treatment options for FND remain elusive. Partly as a result of this, prognosis is poor, ${ }^{6,7}$ symptoms are sometimes found to persist or worsen over time, ${ }^{2}$ and disability resulting from the condition often worse than that seen in organic neurological disease. $^{8}$

Psychological therapy is one of the current mainstays of treatment, with an evidence base for cognitive-behavioural therapy. ${ }^{9,10}$ As well as this, there is promising evidence that many patients benefit from physiotherapy, ${ }^{11,12}$ and a large multicentre randomized controlled trial (RCT) on the
Ther Adv Psychopharmacol 2020, Vol. 10: 1-15 DOI: 10.1177/ 2045125320912125

(c) The Author(s), 2020. Article reuse guidelines: sagepub.com/journalspermissions
Correspondence to: Matthew Butler

The Institute of Psychiatry, Psychology and Neuroscience, King's

College London, London SE5 8AF, UK

South London and Maudsley NHS Foundation Trust, Bethlem Royal Hospital,

Monks Orchard Road,

Beckenham, Kent, UK matthew.butler1@slam. nhs.uk

Mathieu Seynaeve

Timothy R. Nicholson

James Rucker

The Institute of

Psychiatry, Psychology

and Neuroscience, King's

College London, London, UK

South London and Maudsley NHS Foundation Trust, Bethlem Royal Hospital, Monks Orchard Road, Beckenham, Kent, UK

Susannah Pick The Institute of Psychiatry, Psychology and Neuroscience, King's College London, London, UK Richard A. Kanaan The Institute of Psychiatry, Psychology and Neuroscience, King's College London, London, UK Department of Psychiatry, University of Melbourne,

Austin Health, Heidelberg, Australia

Andrew Lees

National Hospita for Neurology and Neurosurgery

University College London, London, UK

Allan H. Young

The Institute of Psychiatry,

Psychology and

Neuroscience, King's

College London, London, UK

Department of

Psychological Medicine,

Institute of Psychiatry,

Psychology and

Neuroscience, King's

College London, London, UK 
efficacy of physiotherapy in functional movement disorder is underway. ${ }^{13}$

There is emerging evidence for brief group psychoeducation for psychogenic nonepileptic seizures (PNES), ${ }^{14}$ repetitive transcranial magnetic stimulation (rTMS) in functional paresis, ${ }^{15}$ and multidisciplinary team rehabilitation in a range of functional neurological symptoms. ${ }^{16}$ It is thought that pharmacological management of FND symptoms is inappropriate. ${ }^{2}$ There remains a pressing need for further research into novel treatment options for FND.

Intimately linked to the lack of treatment options is the lack of clarity regarding the aetiology of FND. Current theories however suggest that aberrant somatic self-representation, the brain perceiving dysfunction in a part of the body despite sensory evidence to the contrary due to abnormal top-down cognitive influence, may underlie symptoms. ${ }^{17}$

In the past two decades, research into the therapeutic potential of psychedelics has re-emerged, and there has been interest in the use of psychedelic compounds in a wide range of neuropsychiatric disorders. Studies have shown that psychedelics have a profound effect on neural activity, and are felt to 'deconstrain' networks in the brain, including those responsible for selfrelated processing. ${ }^{17,18}$

There have been promising early results in recent open-label studies in alcohol dependence, ${ }^{19,20}$ tobacco addiction, ${ }^{21,22}$ obsessive-compulsive disorder, ${ }^{23,24}$ existential anxieties in the context of terminal illness, ${ }^{25-27}$ and unipolar depression. ${ }^{28,29}$ Leading on from this, a further two pilot studies using ayahuasca in major depressive disorder have also been published, ${ }^{30,31}$ and placebo-controlled RCTs are currently underway to investigate psilocybin use in treatment refractory depression. ${ }^{32}$ Importantly, rates of serious adverse events have not been high in any of these studies. ${ }^{28,33}$

Leading on from this, a 2016 review suggested that FND may be an interesting candidate for exploration of potentially efficacious psychedelic therapy. ${ }^{32}$ Furthermore, a 2017 review highlighted the theoretical potential for psychedelics to be explored as a therapeutic option in FND due to their ability to disrupt aberrant self-attentional networks, and suggested that controlled trials of psychedelic therapy in conjunction with physiotherapy or psychotherapy (current best practice) could be considered in for carefully selected patients with FND. ${ }^{17}$

In this article, we describe a systematic review of the literature describing psychedelic treatment in patients with functional neurological disorder to examine whether further clinical research of psychedelic therapy for patients suffering with functional neurological disorders could be considered in future. Due to changing societal attitudes and legal constraints in 1967 resulting in restricted access to psychedelic compounds, there have been almost no modern clinical studies utilizing psychedelic therapy in psychiatric disorders until recently. Because of this, all of the relevant studies included in this review date from an era before the advent of modern clinical trials.

\section{Methods}

The PsycINFO and MEDLINE databases (19402000) were searched using the following terms: 'LSD, lysergic acid diethylamide, psilocybin, psychedelic, hallucinogen, mescaline, ayahuasca AND hysterical, dissociative, conversion, conversion disorder, hysteria, functional neurological disorder, psychogenic'. The 'Multidisciplinary Association for Psychedelic Studies' (MAPS) Psychedelic Bibliography contains a comprehensive overview of psychedelic research, including a complete list of all studies on the therapeutic use of psychedelics from 1931-199534; this database was manually searched for titles or abstracts including the above search terms. Subsequent examination of reference lists identified other potential eligible studies or review articles.

Search results were screened by reading the titles and abstracts by MB and MS. Any discrepancies were resolved through consensus. The full text of screened abstracts was then reviewed to confirm inclusions. Where studies referred to the treatment of multiple patient populations, results of treatment for functional neurological disorder (or equivalent diagnosis) specifically were extracted.

\section{Criteria for study inclusion}

Details of the protocol for this systematic review were registered on PROSPERO (registration number CRD42019118534) and can be accessed at https://www.crd.york.ac.uk/PROSPERO/display_record.php?RecordID $=118534$. 
Table 1. Methodological quality of included studies. ${ }^{a}$

\begin{tabular}{|c|c|c|c|c|c|c|c|}
\hline Study & 1 & 2 & 3 & 4 & 5 & 6 & Overall \\
\hline Sandison et al. $(1954)^{36}$ & - & - & - & - & $+/-$ & - & Low \\
\hline Eisner and Cohen (1958)37 & - & $+/-$ & - & - & - & - & Low \\
\hline Chandler and Hartman (1960)38 & $+/-$ & $+/-$ & - & - & - & + & Low \\
\hline Leuner $(1961)^{39}$ & - & - & - & - & - & - & Low \\
\hline Duché $(1961)^{40}$ & - & $+/-$ & - & - & $+/-$ & - & Low \\
\hline Heyder $(1963)^{41}$ & - & - & - & - & $+/-$ & - & Low \\
\hline Whitaker $(1964)^{42,43}$ & - & - & - & - & - & - & Low \\
\hline Baker $(1967)^{44}$ & - & $+/-$ & - & - & - & + & Low \\
\hline Leuner (1967)45 & - & - & - & - & - & - & Low \\
\hline
\end{tabular}

a1. Is the selection method clear? 2. Was the exposure adequately ascertained? 3 . Was the outcome adequately ascertained? 4. Were other alternative causes that may explain the observation ruled out? 5 . Is the follow up long enough for outcomes to occur? 6. Is the case(s) described with sufficient details to allow other investigators to replicate? ++ , yes; +/-, partially; -, no.

Trials were included if they featured new medically supervised psychedelic drug administration with or without psychotherapy in patients with FND and included outcome data. Papers were excluded if an English or French language copy was not available. Furthermore, review papers and papers with duplicated results were excluded.

\section{Risk of bias (quality) assessment}

The case series and reports were scored using a tool for evaluating the methodological quality of case reports and case series as reported by Murad and colleagues. ${ }^{35}$ This tool is recent and has not been validated; however, to our knowledge there exists no consensus on a rating tool for such studies. Briefly, the case series and reports were ranked on four domains of selection, ascertainment, causality and reporting. Articles were checked by $\mathrm{MB}$ and $\mathrm{TN}$, and any discrepancies were resolved through consensus. An overall judgement of methodological quality was then made based on the extracted information, and studies were given a rating of low, moderate, or high (summarized in Table 1).

\section{Outcome measures}

Patients were classified as either not improved, improved, or recovered based on qualitative descriptions of outcomes in each study. Where it was not clear if a patient made improvement or did not recover, they were classed as not improved. Qualitative descriptions of adverse events were included as a second primary outcome.

\section{Results}

A total of 735 papers were identified through electronic records and other sources, representing 705 papers after removal of duplicates. Of these, 678 were excluded after screening of the titles or abstracts, leaving 27 that were screened in more detail for eligibility. Of these, 18 were excluded to leave the nine papers used in this systematic review. Study selection is illustrated in Figure 1.

The literature on the use of psychedelic therapy in the treatment of FND is summarized in Table 2. A total of nine studies published between 1954 and 1967 were included, encompassing the period prior to the prohibitions of LSD in the late 1960s; to the best of our knowledge, no modern studies with original results have been published in this area. In most cases, patients had psychotherapy alongside psychedelic drug administration in what became known as 'psycholytic therapy' (in some cases, larger doses were also used in 'psychedelic therapy').

Sample sizes ranged from 1 to 6 patients, giving an aggregate sample of 26 across the included studies. 


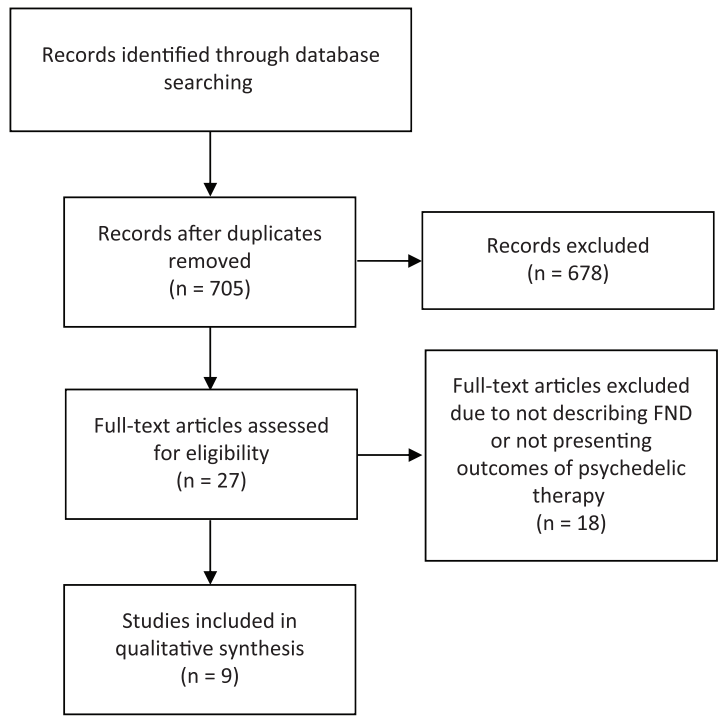

Figure 1. Study selection. FND, functional neurological disorder.

LSD was the most commonly used psychedelic, with some studies also using psilocybin or mescaline. The number of therapeutic sessions of psychotherapy $+/-$ psychedelic substance administration ranged from 1 to 26, with descriptions of therapeutic methods used widely varying, and in some cases not described in any detail. Psychedelics were given either orally or intramuscularly, with doses of LSD ranging from 25 to $2000 \mu \mathrm{g}$, and doses of psilocybin ranging from 3 to $15 \mathrm{mg}$.

Of the 26 patients described with FND, 69\% $(n=18)$ were found to have made at least some recovery, with $23 \%(n=6)$ having recovered completely. The study authors were mixed in their recommendations for psychedelic therapy in patients with FND.

In every case, the methodological quality of included papers was rated as poor.

\section{Description of studies}

Sandison et al. 1954 and 1957. Lysergic acid diethylamide (LSD) was first synthesized in 1938, and by 1957 was being marketed by the pharmaceutical company Sandoz. ${ }^{46}$ In 1954, British psychiatrist and psychotherapist Ronald Sandison and colleagues published their findings after administering LSD to patients with psychoneurotic disorders, including four patients with 'conversion hysteria', at Powick Mental Hospital near Worcester. Their patients were given psychedelics and received psychodynamic psychotherapy during the therapeutic sessions, a common method throughout the studies included in this review. In this case, psychotherapy plus $25 \mu \mathrm{g}$ of oral LSD (which was uptitrated to a maximum of $400 \mu \mathrm{g}$ depending on response) was delivered to patients approximately weekly. There was no control group, a further common theme to the studies described in this review.

One patient with 'conversion hysteria' recovered after nine sessions of treatment, and one patient showed moderate recovery after three sessions. Another patient, still undergoing treatment at the time, had improved after 16 sessions, and another had 'refused after one treatment'. This fourth patient dropped out after one session, describing the 'intensely unpleasant' effect of LSD as 'the most soul-searing experience I have ever had' (p. 502). Other recorded side effects, all of which were transient and related to the acute psychedelic state, were facial flushing, rapid or distressed breathing, disturbed or violent behaviour, panic attacks, and depersonalization.

With these mixed findings, Sandison et al. concluded that, of LSD psychotherapy, 'conversion hysterics appear to do badly, but these cases were treated early in our series and the amount of treatment given may have been insufficient' (p.502) They also noted that 'all of our early cases were either severe obsessional neurotics with a bad prognosis or were patients who had been ill for a considerable time and who had previously had prolonged treatment either by psychotherapy or other means without improvement' (p.501). ${ }^{36}$

In his 1957 follow up (not included in Table 2), Sandison commented on 12 patients with 'hysteria (all forms)': 1 had recovered, 2 had greatly improved, 4 had moderately improved, and 5 had not improved. Sandison included patients from the 1954 study as well as new patients, and he did not clearly specify the origin of each patient, rendering this follow up unsuitable for further analysis. ${ }^{47}$

At a psychedelic symposium held in 1960, Sandison coined the term 'psycholytic' therapy to describe psychotherapy with patients under the influence of psychedelics. ${ }^{48} \mathrm{He}$ felt that psychedelics allowed individuals to relive childhood experiences through loosening of ego-defences. Psycholytic therapy used relatively low doses of LSD or psilocybin, plus psychotherapy, delivered over numerous sessions. This is in contrast to 


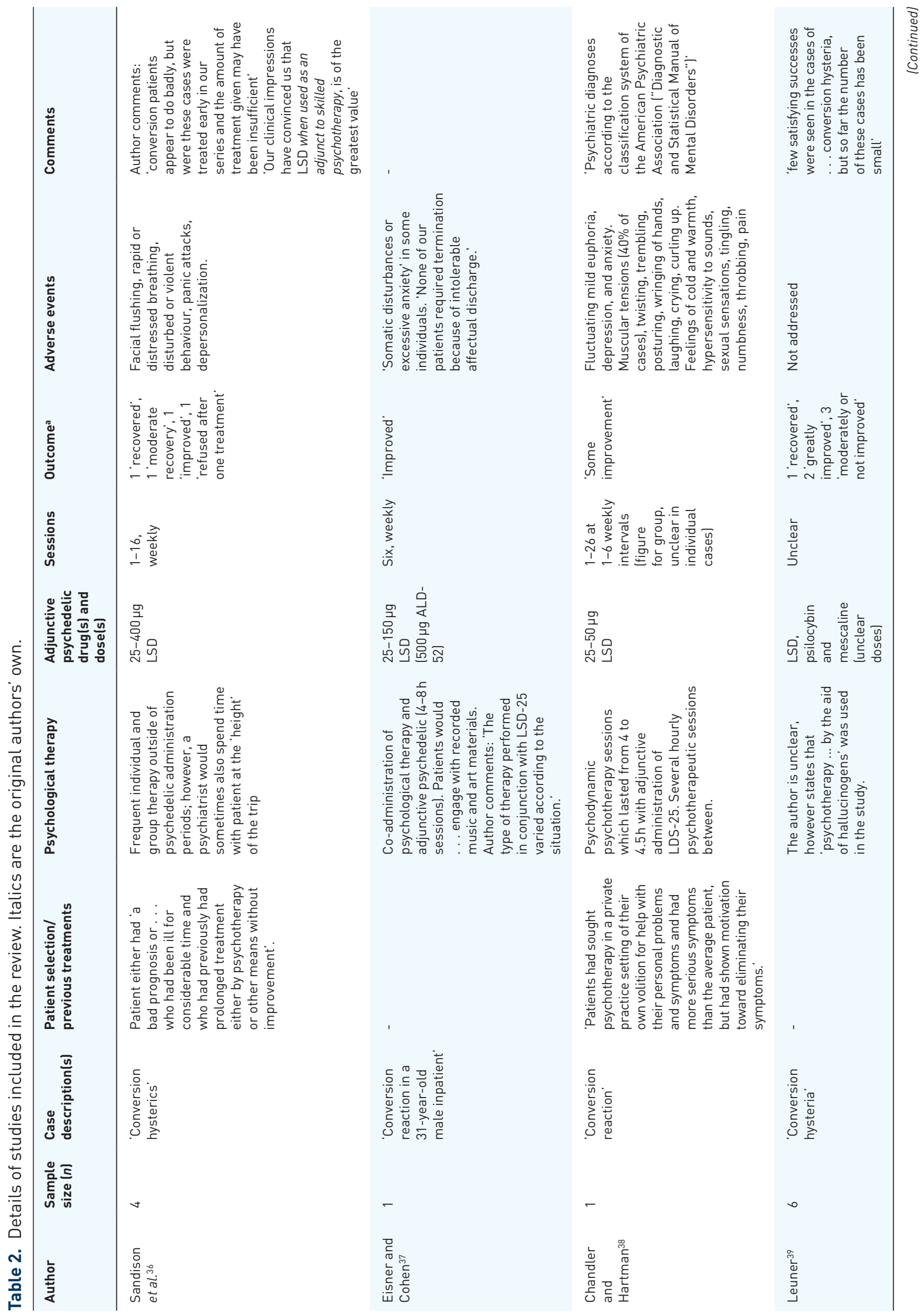




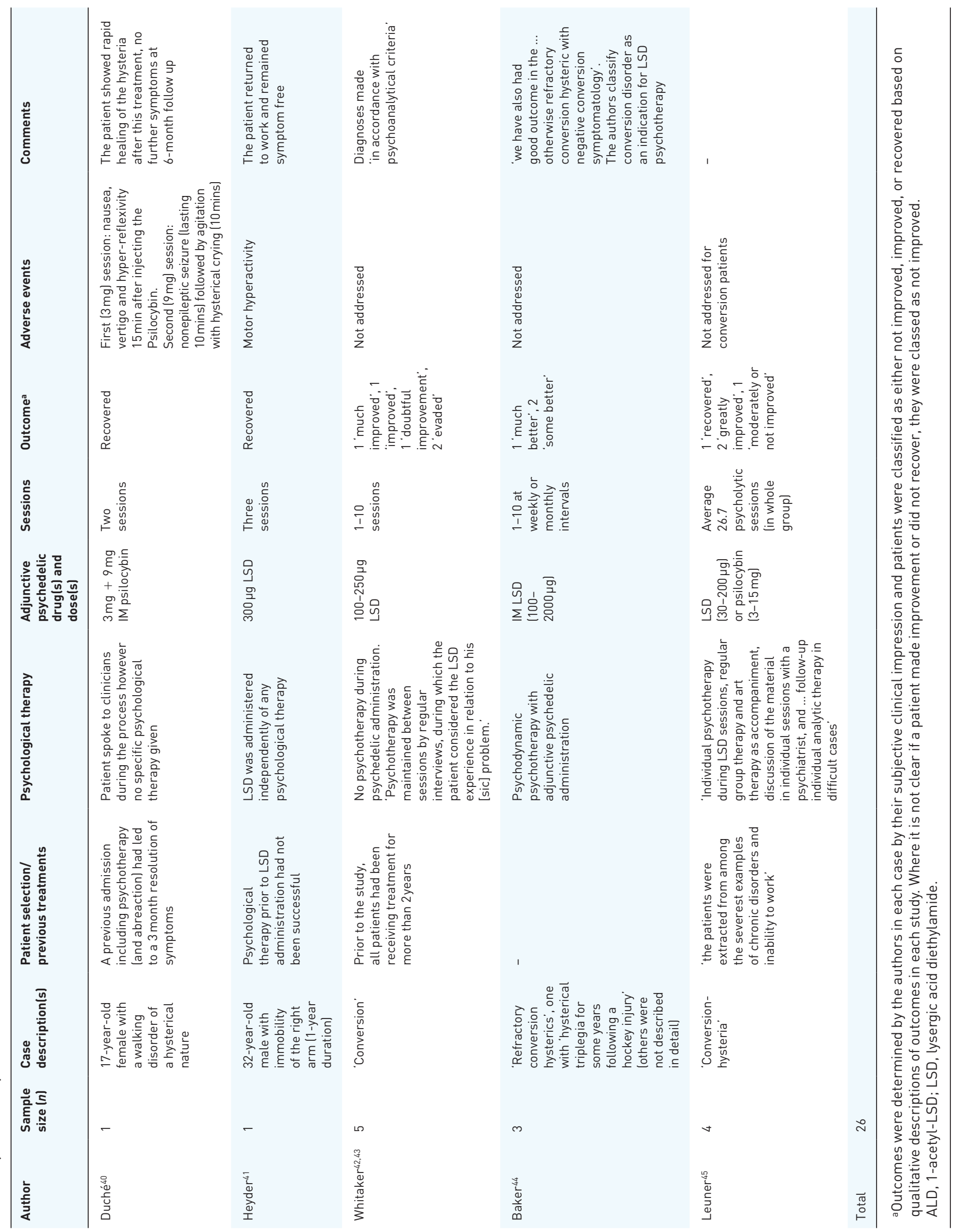


'psychedelic' therapy, in which much larger doses were used less frequently to elicit ineffable, mystical experiences that were thought to have therapeutic potential.

Eisner and Cohen 1958. A 1958 study by Betty Eisner and Sidney Cohen at the Veterans Administration Center in Los Angeles included one 31-year-old male inpatient with 'conversion reaction'. Six sessions of LSD psycholytic therapy with two therapists present every week was given to this patient, with the dose of LSD started at $25 \mu \mathrm{g}$ and increased to a maximum of $150 \mu \mathrm{g}$, or $500 \mu \mathrm{g}$ of 1-acetyl-LSD (ALD-52; a chemical analogue of LSD, with this dose equivalent to 200-250 $\mu \mathrm{g}$ LSD). The therapy sessions lasted $4-8 \mathrm{~h}$, the patient lying on a couch with music playing. Art materials were used at the end of the session to assist with self-expression and therapeutic integration.

Improvement was determined by the two doctors present in the sessions, the patient, and the 'person closest to him', with follow up interviews after $6-17$ months. The patient was found to have 'improved' with 'the important criteria of benefit' being 'continuing success in behavioural adaption'. ${ }^{37}$

Giberti and Gregoretti 1959. In an Italian study (not included in Table 2), Franco Giberti and Luciano Gregoretti compared 12 patients with 'conversion disorder' in 2-8 weekly sessions of psycholytic therapy with $100 \mu \mathrm{g}$ LSD and 1-2 weekly sessions with $500 \mu \mathrm{g}$ of LAE-32 (another LSD analogue that is weaker and shorter-lasting) with 14 patients with obsessive compulsive disorder (OCD). During the dosing sessions, the patients with 'conversion disorder' showed amplification of sensory and motor impairment; however, in some cases showed 'miraculous' improvement. ${ }^{49}$ Unfortunately, the authors did not provide outcome data for the patients, rendering this follow up unsuitable for further analysis.

Chandler and Hartman 1960. Arthur Chandler and Mortimer Hartman describe their Beverly Hills study of LSD psychotherapy with an average of six sessions of 4-h therapy at intervals of 1-6 weeks (average 2 weeks). Their patient group (a mixed psychiatric group) had all previously sought therapy and therefore 'showed at least a modicum of insight and motivation,' according to the authors.
The usual starting dose was 25 or $50 \mu \mathrm{g}$ of LSD, given orally. The dose was increased by $25-50 \mu \mathrm{g}$ each session to a maximum of $150 \mu \mathrm{g}$. The therapy was delivered in 'carpeted and furnished rooms' and the patient was asked to lie back with their eyes closed. As with Eisner and Cohen, music (classical music in this case) was used as an accompaniment. The one patient in this study with conversion disorder was reported to have made 'some improvement' by the end of the sessions. ${ }^{38}$

Although not specific to the FND patients, Chandler and Hartman were one of the few authors who commented on side-effects. They noted that 'risks involved are related to the psychological and not to the pharmacologic effects of the drug' and that fluctuating feelings of wellbeing, mild euphoria, depression, and anxiety were the commonest difficulties following LSD sessions.

Also noted during the sessions were varying degrees of somatic symptoms (present in $70-75 \%$ of patients in at least one session) including feelings of cold and warmth, hypersensitivity to sounds, general sensuous feelings of well-being throughout the body, sexual sensations, various paraesthesias, such as tingling, numberless, and throbbing, and, lastly, pain (frequent occurrence). Muscular tension was also seen in $40 \%$ of cases, and they also noted twisting, trembling, posturing, wringing of hands, laughing, crying, curling up into a foetal position.

Ling and Buckman 1960. A 1960 paper by Thomas Ling and John Buckman described 50 patients, two of which had conversion hysteria. Most of the patients were described as 'improving'; however, the authors did not stratify the group based on diagnosis, and therefore we do not know the outcome for the two patients with conversion disorder, and for this reason the study is not analysed further. The authors did, however, state that monosymptomatic conversion symptoms were a contraindication for LSD therapy due to their presumed poorer outcomes relative to other patient groups. ${ }^{50}$

Leuner 1961. In his 1961 report, Hanscarl Leuner describes giving LSD, psilocybin and mescaline to a cohort of patients at the Hospital for Mental Diseases at Göttingen University, Germany, of which six were described as having 'conversion hysteria'. Of those six, one 'recovered', two 'greatly improved' and three improved 'moderately or [did] not improve'. The paper does not go into any 
detail about how the therapy was carried out and does not define the doses used. Leuner is cautious when interpreting his findings with conversion disorder, writing that 'few satisfying successes were seen in the cases of . . conversion hysteria, but so far the number of these cases has been small' (p. 68). ${ }^{39}$

Duché 1961. In a 1961 case report, French doctor DJ Duché described a single session of psycholytic therapy with $3 \mathrm{mg}$ and then $9 \mathrm{mg}$ of intramuscular (IM) psilocybin in a 17-year-old girl with 'a walking disorder of a hysterical nature' which she had suffered with since the age of 12 in the context of a difficult childhood.

The first $(3 \mathrm{mg})$ session resulted in nausea, vertigo and hyper-reflexivity $15 \mathrm{~min}$ after injecting the Psilocybin. The $9 \mathrm{mg}$ session started with a nonepileptic seizure, lasting $10 \mathrm{~min}$, followed by $10 \mathrm{~min}$ of agitation with hysterical crying; she denied nausea or vertigo at this stage. This was then followed by euphoria with disorientation and hallucinations about family members (the author does not mention how long this lasted). At the end of the session, the girl was able to 'visually relive some traumatic episodes of her life,' and was noted to have 'rapid healing of the hysteria after this treatment' (p.3062) which persisted at 6-month follow up. ${ }^{40}$

Heyder 1963. In a further case report, Dietrich Heyder describes the case of a 32-year-old man who presented to Norfolk Mental Health Center in Virginia with functional immobility of the right arm for 1 year secondary to an industrial accident (a welding torch explosion). He was given $300 \mu \mathrm{g}$ of LSD plus psychotherapy three times within 8 days. He left after the third session able to use his right arm completely freely. He was then described as going fishing 2 days later and 'his picture appeared in the paper for catching the biggest fish of the season' (p.369). He subsequently returned to work and remained symptom free. ${ }^{41}$

Whitaker 1964. The Australian psychiatrist Howard Whitaker described his study at the Royal Women's Hospital in Melbourne. ${ }^{42}$ The study included 100 patients given $100-250 \mu$ g of LSD and was the first described in this review to include outcome data versus a control group. There were a total of five patients in the study who were described as suffering with 'conversion'. The average number of LSD-plus-psychotherapy sessions was 3.28. Whitaker described the five patients as follows; one 'much improved', one 'improved', one showed 'doubtful improvement', and two 'evaded'. ${ }^{43}$

Baker 1967. A 1967 paper by Edward Baker describes his treatment of 150 patients at Toronto Hospital between 1961 and 1964 using LSD psychotherapy given several weeks or months apart. The patients were given 1-10 courses of intramuscular (IM) LSD (100-2,000 $\mu \mathrm{g})$ plus psychotherapy at weekly or monthly intervals. Three patients were described as 'refractory conversion hysterics'. After the therapy had been completed, one of these patient was described as 'much better', and the remaining two were 'some better', which the author described as a 'good outcome'. He went on to classify 'conversion disorder' as an indication for LSD psychotherapy.

$\mathrm{He}$ describes the case of one particular patient with conversion disorder as follows; 'This man had been a hysterical triplegic for some years following a hockey injury. Earlier LSD psychotherapeutic interview recovered his two limbs to his use. His overdetermined hysterical conversion represented 'mental amputation of the leg' at mid-thigh. The state did not budge with LSD doses to $1600 \mu \mathrm{g}$. A final dose of $2000 \mu \mathrm{g}$ LSD recovered the sensory motor and integrative use of this limb within $10 \mathrm{~min}$ of injection. This man has remained well over 2 years' (p. 196). ${ }^{44}$

Leuner 1967. In 1967, Leuner again describes the results of a follow-up study of LSD psycholytic therapy on 82 patients at the Hospital for Mental Diseases at Göttingen University, Germany. The study included four patients with 'conversionhysteria', of whom; one 'recovered (able to work)', two were 'greatly improved', and one was 'moderately or not improved (unable to work)'. ${ }^{45}$

Soskin 1973. A 1973 paper by Robert Soskin (not included in Table 2) describes giving LSD $(n=14)$ and placebo $(n=14)$ to patients admitted to the Topeka Veterans' Administration Hospital in Kansas. Unfortunately, Soskin does not categorize the patients based on their diagnosis; however, an averaged 'Conversion Hysteria' score on the Wittenborn Psychiatric Rating Scale was utilized. The patients had twice-weekly psychotherapy for a total of 13 weeks; a total of five of these sessions were accompanied by oral LSD administration $(50-300 \mu \mathrm{g})$ in the LSD group. The placebo group received a combination of $25 \mathrm{mg}$ of chlordiazepoxide and $25 \mathrm{mg}$ of methylphenidate in each of 
their drug sessions. Conversion Hysteria score was found to decrease from 4.89 to 3.50 in the LSD group and from 5.82 to 4.96 in the control group. This effect was not significant for the LSD versus control group $(\mathrm{F}=1.31)$; however, it was significant in the pre versus post scores $(t=3.45$, $p<0.01) .{ }^{51}$ Due to the omission of specific patient details, this study was not included for further analysis.

\section{Discussion}

In this review, we systematically analysed papers on the use of psychedelics in the treatment of 'conversion' and 'hysteria' conditions. All studies included were from the era prior to the legal restrictions of psychedelics in the late 1960s. Nine studies were included for outcome analysis, in which over two-thirds of included patients were judged to have made at least some recovery with psychedelic plus psychotherapy treatment (summarised in Table 2).

The included studies utilized either the psycholytic paradigm, which incorporated low doses of adjunctive psychedelics as part of psychotherapy (almost always psychoanalytically themed). This aimed to use the psychedelics as part of an attempt to explore unconscious material in the therapy. Other studies utilised 'psychedelic' therapy, which involved giving large doses of psychedelics outside of a psychotherapy session, with sessions before or afterwards to explore the psychedelic experience..$^{52}$ As noted by Leuner (1967) 'it is . . best that we distinguish clearly between these two methods; that is, between psycholytic therapy on the one hand and psychedelic therapy on the other. All they have in common is the use of the same drugs.' Due to the patchy reporting of methodology and relatively low numbers of total participants, it is difficult for us to conclude that one particular method was superior to any other, or indeed which aspect(s) of the multifaceted therapies was responsible for the clinician-rated improvements.

It proved difficult to tease out from these studies the degree to which the psychotherapy given influenced the outcomes. As Soskin summarizes in his paper: 'It is particularly difficult to evaluate the role which LSD plays in the treatment process. Since an average LSD session lasts from 8 to 12 hours, the therapist is making an unusual investment of time and attention. This degree of therapist involvement could certainly help the patient to feel that he is cared for in a special way and could be a more important determinant in contributing toward improvement than the action of the drug' (p.411). ${ }^{51}$ Due to the limited descriptions of the psychotherapy given, it remains to be seen whether the form, quantity or frequency of psychotherapy made a qualitative difference to the doctors' or patients' impression of how they improved.

Whilst almost all of the studies included in this review used LSD, modern therapeutic psychedelic studies have tended instead to use psilocybin. Psilocybin has similar psychological and physiological effects to LSD; however, has a shorter halflife (and hence duration of action) and therefore allows for therapeutic sessions to be delivered on a day-case basis, thus avoiding the cost of overnight stays. As well as this, anecdotal evidence has pointed towards psilocybin having less of a capacity to produce prolonged distressing bad trips. ${ }^{33}$ Psilocybin is also now being manufactured to Good Manufacturing Practice (GMP) standard by a UK-based pharmaceutical company (Compass Pathways Ltd., Altrincham, Cheshire, UK).

\section{Theoretical considerations of therapeutic mechanisms}

Contemporary theories of FND suggest aberrant neural self-representation may lead to symptoms. ${ }^{53-56}$ Such models suggest that positive and negative motor and sensory symptoms arise through altered awareness and attentional allocation to somatic and affective self-representations; specifically, abnormal body-focussed attention arises as a result of an over-reliance on internally generated predictions of somatic representation at the expense of sensory input, leading to errors in interpretation of sensory data (in other words, a mismatch between predictive models of what the body is doing in comparison with what it is actually doing). ${ }^{57}$

It is postulated that a wide-range of factors may lead to heightened self-monitoring, ${ }^{58}$ leading to distorted representation of somatic states and an abnormally high attentional diversion to the body. ${ }^{59}$ Indeed, people who scored highly on the Somatoform Dissociation Questionnaire (SDQ20) have been shown to have a propensity to focus attention on the self, leading to a higher than average awareness of the body and somatic signals. ${ }^{59}$ Taken together, these results support theories that FND symptoms may arise due to an alteration in neural representation of the somatic self. 60 
Neurobiological theories of FND have been supported by neuroimaging in patients with motor functional neurological disorder and PNES. ${ }^{61,62}$ fMRI studies have found increased functional connectivity in patients with functional neurological symptoms the ventromedial prefrontal cortex, precuneus and insula, which comprise the default mode network (DMN), a neural system associated with the 'sense of self' ${ }^{60,63-65} \mathrm{DMN}$ activity is increased in tasks which involve representation of the self, including somatic self-representation and retrieval of self-imagery. ${ }^{17,66}$

$5-\mathrm{HT}_{2 \mathrm{~A}}$ receptors are densely distributed in neural networks such as the $\mathrm{DMN}^{67}$ and the $5-\mathrm{HT}_{2 \mathrm{~A}}$ agonism of psychedelics have been shown in fMRI studies to reduce the functional connectivity (i.e. increase the entropy) of neural networks such as the DMN. ${ }^{18}$ Strikingly, the extent of reduction in connectivity correlates with selfreported degree of loss of selfhood ('ego-dissolution'), which adds weight to the hypothesis that this network is responsible for self-related cognition. ${ }^{18}$

\section{Safety}

Psychedelic substances have been shown consistently to be physiologically nontoxic relative to other psychoactive drugs (as well as being nonaddictive), and notably safe when given in medically controlled and psychologically supportive settings. ${ }^{32,68,69}$ In an analysis of harms to user and society of recreational psychoactive substances, LSD and psilocybin were ranked as amongst the least harmful. ${ }^{69}$ As well as this, a population study found no increase in mental health problems in those who self-reported psychedelic use..$^{70}$ Despite this, there is some lack of clarity; for example, one 2019 anonymous survey of university students founds increase in self-reported mental health problems in users of psychedelics. ${ }^{71}$

The toxicity ratio (i.e. the ratio between the therapeutic and estimated lethal dose) is thought to be 1000 or more for both LSD and psilocybin. This is in contrast to many other prescribed medications and illicit drugs, which often feature much smaller toxicity ratios. ${ }^{68}$ The risks associated with the medically supervised use of psychedelics is thought to be low, and guidelines for their safe use in research have been published based on phase I and II trials. ${ }^{72}$ Large-scale population studies have in fact shown that the use of psychedelics is associated with lower relative risk of suicide, ${ }^{73}$ and psychological stress and need for psychiatric medicine. ${ }^{74}$

Adverse effects of psychedelic administration, if any, often went unreported in the studies included in this review. Many of the reported adverse effects were mild and transient; however, some more serious events such as depersonalization were also experienced. The profound consciousness-altering effects of psychedelics, particularly LSD, can induce a degree of anxiety or panic which some of the patients included in this study may have found distressing; there was one notable account of a patient, who described the experience as 'the most soul-searing experience I have ever had'. ${ }^{36}$ We also noted that in a few cases from the patients included in the studies with other psychiatric conditions than FND (i.e. not discussed in this review), the authors decided to terminate the LSD trip due to a negative psychological reaction.

Despite the evidence for the safety of psychedelics that has emerged, there are particular considerations that must be taken account in the FND population, who feature motor or sensory symptoms. As noted from the included studies, psychedelics sometimes produce abnormal physical and motor effects, such as, for example, muscular tension and numbness. The young woman in the study by Duché (1961) also had a nonepileptic seizure after the second administration of IM psilocybin. ${ }^{40}$ Although no patients in this review were described as worsening, it is of course possible that psychedelics have the potential to exacerbate FND symptoms.

\section{Limitations}

There are many limitations to this review, and the results should be interpreted with caution. One key limitation of this review is the generally poor quality of the 'pre-prohibition' studies by today's standards. Outcome measures were typically limited to clinician-rated 'improvement' versus nonimprovement, and no explanation to validate these observations is offered in any of the studies we reviewed. It is also unclear in many cases whether 'improvement' represented symptom improvement, or rather patients becoming more accepting of their symptoms even without improvement.

We were unable to generate a pooled effect size due to inconsistent outcome measures and lack of 
comparative effect size measurements between studies, and therefore could not undertake metaanalysis. Furthermore, almost all of the trials included lacked a control group, and their assessment procedures were poorly described. The evidence presented was often personal and anecdotal, and two case studies formed part of the analysis. Overall, the likelihood of bias is substantial.

Although many of the studies included patients over periods over weeks to months, follow up data was rarely included. Based on this lack of data, we were in most cases unable to conclude whether any improvements noted by the authors were sustained longer term. A notable exception is the case report by Duché (1961), which showed continuing symptom resolution at 6 months.

It is likely that selection biases were incorporated in these studies, with authors often choosing patients who had not responded to ongoing psychological therapy or whom were motivated to engage, for example, Chandler and Hartman explained that their patients had all previously sought therapy and therefore 'showed at least a modicum of insight and motivation'. ${ }^{38}$

In the included studies, multiple types of psychedelics were used (LSD, psilocybin, mescaline, and LSD analogues), and the range of doses used in the individual studies varied widely. Although these compounds have distinct molecular structures, they all exert their effects through agonism of $5-\mathrm{HT}_{2 \mathrm{~A}}$ receptors. ${ }^{75}$ When specified, except from in some preliminary test administrations, significant doses were used, and, when not specified we have no reason to suspect unusually low (or high doses) would have been used. Specifically for LSD, patients received smaller doses of $25 \mu \mathrm{g}$ (which would have been unlikely to produce any psychedelic effect) to doses of up to $2000 \mu \mathrm{g}$, far eclipsing those used in more modern studies. This, coupled with the heterogeneity of concurrent psychological intervention in the studies, makes it more difficult to draw a conclusion regarding a common therapeutic mechanism of psychedelic drugs in FND from this review.

All studies included in this review were published in the period prior to the widespread use of modern standardized diagnostic manuals, such as the Diagnostic and Statistical Manual of Mental Disorders (DSM), ${ }^{76}$ meaning that there is likely significant heterogeneity in the presentations, which may not have reached today's diagnostic criteria for FND. Most included patients were diagnosed with now outdated terms such as 'conversion hysteria', or 'conversion reaction', which we recognise have historically been protean diagnoses. Where authors described specific symptoms of their 'conversion' patients, these were included; however, in many cases this information is missing and it is likely that the patient population included in this review had clinical presentations that diverge to some degree from modern presentations. Indeed, one review of historical studies found calculated significant (29\% in the 1960 s and $17 \%$ in the 1960 s) misdiagnosis in patients with unexplained sensory and motor symptoms. ${ }^{77}$ This represents a clear limitation in generalizing the results of the studies included in this review to modern FND populations.

\section{Conclusion}

In this review, we have summarised preprohibition literature describing the use of psychedelic psychotherapy in FND. The results from preprohibition literature, whilst poorly designed in comparison with modern standards, indicate that therapy was safe and mostly well tolerated by patients, with some improvements on subjective clinician-rated standards.

Overall, we conclude that there are encouraging lessons to learn from preprohibition studies of adjunctive psychedelic therapy in functional neurological disorder, and that further investigations of feasibility and safety of treatment with psychedelics (more specifically, psilocybin) in patients with functional neurological disorders is a potential future research option. We recommend that preliminary patient liaison and acceptability work is undertaken before psychedelics trials are considered in patients with FND.

\section{Funding}

The authors disclosed receipt of the following financial support for the research, authorship, and/or publication of this article: This report is independent research supported by the UK National Institute for Health Research (NIHR) via Clinician Scientist Fellowship Awards for both JR (CS-2017-17-007) and TN (CS-201414-016). This study presents independent research part-funded by the National Institute for Health Research (NIHR) Biomedical Research 
Centre at South London and Maudsley NHS Foundation Trust and King's College London. The views expressed in this publication are those of the author(s) and not necessarily those of the NHS, the National Institute for Health Research or the Department of Health.

\section{Conflict of interest statement}

King's College London receives grant funding for phase I and II trials with psilocybin, led by JR and AY, from Compass Pathways Ltd. JR has attended trial related meetings paid for by Compass Pathways Ltd. Compass Pathways Ltd. had no influence over the inception, design, execution or publication of this work.

\section{ORCID iDs} Matthew Butler (D) https://orcid.org/0000-0002-
9734-6539

\section{Allan H Young (iD https://orcid.org/0000-0003- 2291-6952}

James Rucker https://orcid.org/0000-00034647-8088

\section{References}

1. Ahmad $\mathrm{O}$ and Ahmad KE. Functional neurological disorders in outpatient practice: an Australian cohort. $\mathcal{F}$ Clin Neurosci 2016; 28: 93-96.

2. Espay AJ, Aybek S, Carson A, et al. Current concepts in diagnosis and treatment of functional neurological disorders. FAMA Neurol 2018; 75: 1132-1141.

3. Stone J, Carson A, Duncan R, et al. Who is referred to neurology clinics? - The diagnoses made in 3781 new patients. Clin Neurol Neurosurg 2010; 112: 747-751.

4. Carson A and Lehn A. Epidemiology. Handb Clin Neurol 2016; 139: 47-60.

5. Vedat Sar, Akyuz G, Kundakçı T, et al. Childhood trauma, dissociation, and psychiatric comorbidity in patients with conversion disorder. Am F Psychiatry 2004; 161: 2271-2276.

6. Kanaan RA, Armstrong D and Wessely SC. Neurologists' understanding and management of conversion disorder. $\mathcal{F}$ Neurol Neurosurg Psychiatry 2011; 82: 961-966.

7. Gelauff J, Stone J, Edwards M, et al. The prognosis of functional (psychogenic) motor symptoms: a systematic review. $\mathcal{F}$ Neurol Neurosurg Psychiatry 2014; 85: 220-226.
8. Carson A, Stone J, Hibberd C, et al. Disability, distress and unemployment in neurology outpatients with symptoms "unexplained by organic disease." F Neurol Neurosurg Psychiatry. 2011; 82: 810-813.

9. Goldstein LH, Chalder T, Chigwedere C, et al. Cognitive-behavioral therapy for psychogenic nonepileptic seizures: a pilot RCT. Neurology 2010; 74: 1986-1994.

10. LaFrance WC, Baird GL, Barry JJ, et al. Multicenter pilot treatment trial for psychogenic nonepileptic seizures: A randomized clinical trial. fAMA Psychiatry 2014; 71: 997-1005.

11. Nielsen G, Buszewicz M, Stevenson F, et al. Randomised feasibility study of physiotherapy for patients with functional motor symptoms. $\mathcal{F}$ Neurol Neurosurg Psychiatry. 2017; 88: 484-490.

12. Jordbru AA, Smedstad LM, Klungsøyr O, et al. Psychogenic gait disorder: a randomized controlled trial of physical rehabilitation with one-year follow-up. $\mathcal{F}$ Rehabil Med 2014; 46: 181-187.

13. Nielsen G, Stone J, Buszewicz M, et al. Physio4FMD: Protocol for a multicentre randomised controlled trial of specialist physiotherapy for functional motor disorder. BMC Neurol 2019; 19: 242.

14. Chen DK, Maheshwari A, Franks R, et al. Brief group psychoeducation for psychogenic nonepileptic seizures: a neurologist-initiated program in an epilepsy center. Epilepsia 2014; 55: 156-166.

15. Broersma M, Koops EA, Vroomen PC, et al. Can repetitive transcranial magnetic stimulation increase muscle strength in functional neurological paresis? A proof-of-principle study. Eur $\mathcal{F}$ Neurol 2015; 22: 866-873.

16. Demartini B, Batla A, Petrochilos P, et al. Multidisciplinary treatment for functional neurological symptoms: a prospective study. f Neurol 2014; 261: 2370-2377.

17. Bryson A, Carter O, Norman T, et al. 5-HT2Aagonists: a novel therapy for functional neurological disorders? Int $\mathcal{F}$ Neuropsychopharmacol 2017; 20: 422-427.

18. Carhart-Harris RL, Muthukumaraswamy S, Roseman L, et al. Neural correlates of the LSD experience revealed by multimodal neuroimaging. Proc Natl Acad Sci U S A 2016; 113: 4853-4858.

19. Krebs TS and Johansen PØ. Lysergic acid diethylamide (LSD) for alcoholism: meta-analysis of randomized controlled trials. F Psychopharmacol 2012; 26: 994-1002. 
20. Bogenschutz MP, Forcehimes AA, Pommy JA, et al. Psilocybin-assisted treatment for alcohol dependence: a proof-of-concept study. J Psychopharmacol 2015; 29: 289-299.

21. Johnson MW, Garcia-Romeu A and Griffiths RR. Long-term follow-up of psilocybin-facilitated smoking cessation. Am $\mathcal{F}$ Drug Alcohol Abuse 2017; 43: 55-60.

22. Johnson MW, Garcia-Romeu A, Cosimano MP, et al. Pilot study of the 5-HT2AR agonist psilocybin in the treatment of tobacco addiction. f Psychopharmacol 2014; 28: 983-992.

23. Castaneda AE, Tuulio-Henriksson A, Marttunen $\mathrm{M}$, et al. A review on cognitive impairments in depressive and anxiety disorders with a focus on young adults. F Affect Disord 2008; 106: 1-27.

24. Moreno FA, Wiegand CB, Taitano EK, et al. Safety, tolerability, and efficacy of psilocybin in 9 patients with obsessive-compulsive disorder. f Clin Psychiatry 2006; 67: 1735-1740.

25. Grob CS, Danforth AL, Chopra GS, et al. Pilot study of psilocybin treatment for anxiety in patients with advanced-stage cancer. Arch Gen Psychiatry 2011; 68: 71-78.

26. Ross S, Bossis A, Guss J, et al. Rapid and sustained symptom reduction following psilocybin treatment for anxiety and depression in patients with life-threatening cancer: a randomized controlled trial. F Psychopharmacol 2016; 30: 1165-1180.

27. Griffiths RR, Johnson MW, Carducci MA, et al. Psilocybin produces substantial and sustained decreases in depression and anxiety in patients with life-threatening cancer: a randomized double-blind trial. F Psychopharmacol 2016; 30: 1181-1197.

28. Carhart-Harris RL, Bolstridge M, Day CMJ, et al. Psilocybin with psychological support for treatment-resistant depression: six-month follow-up. Psychopharmacology (Berl) 2018; 235: 399-408.

29. Carhart-Harris RL, Bolstridge M, Rucker J, et al. Psilocybin with psychological support for treatmentresistant depression: an open-label feasibility study. Lancet Psychiatry 2016; 3: 619-627.

30. de Osório FL, Sanches RF, Macedo LR, et al. Antidepressant effects of a single dose of ayahuasca in patients with recurrent depression: a preliminary report. Rev Bras Psiquiatr 2015; 37: 13-20.

31. Sanches RF, De Lima Osório F, Santos RGD, et al. Antidepressant effects of a single dose of ayahuasca in patients with recurrent depression: a SPECT study. F Clin Psychopharmacol 2016; 36: $77-81$.
32. Rucker JJH, Iliff J and Nutt DJ. Psychiatry \& the psychedelic drugs. Past, present $\&$ future. Neuropharmacology 2018; 142: 200-218.

33. Rucker JJH, Jelen LA, Flynn S, et al. Psychedelics in the treatment of unipolar mood disorders: a systematic review. F Psychopharmacol 2016; 30: 1220-1229.

34. MAPS. Psychedelic Bibliography [Internet], https://maps.org/ (2018, accessed 11 November 2018).

35. Murad MH, Sultan S, Haffar S, et al. Methodological quality and synthesis of case series and case reports. BMF Evid Based Med 2018; 23: 60-63.

36. Sandison R, Spencer A and Whitelaw J. The therapeutic value of lysergic acid diethylamide in mental illness. F Ment Sci 1954; 100: 491-507.

37. Eisner B and Cohen S. Psychotherapy with lysergic acid diethylamide. $\mathcal{F}$ Ment Nerv Dis 1958 ; 127: 528-539.

38. Chandler AL and Hartman MA. Lysergic acid diethylamide (LSD-25) as a Facilitating agent in psychotherapy. AMA Arch Gen Psychiatry 1960; 2: 286-299.

39. Leuner H. Psychotherapy with hallucinogens: a clinical report with special reference to the revival of emotional phases of childhood. In: Crocket R, Sandison RA and Alexander W (eds) Hallucinogenic drugs and their psychotherapeutic use. London: K. K. Lewis \& Co. Ltd., 1961, pp.67-73.

40. Duché DJ. Les Effets de la Psilocybine Dans un Cas d'Hystérie. Sem Hop 1961; 37: 3061-3062.

41. Heyder DW. LSD-25 in Conversion Reaction. Am F Psychiatry 1963; 120: 396-397.

42. Whitaker LH. Lysergic acid diethylamide in psychotherapy. Part I: Clinical aspects. Med $\mathcal{F}$ Aust 1964; 1: 5-8.

43. Whitaker L. Lysergic acid diethylamide in psychotherapy. Med F Aust 1964; 1: 36-41.

44. Baker EFW. LSD psychotherapy; LSD psychoexploration: three reports. In: Abramson HA (ed.) The use of LSD in psychotherapy and alcoholism. Indianapolis, IN: Bobbs-Merrill, 1967, pp.191-207.

45. Leuner H. Present state of psycholytic therapy and its possibilities. In Abramson A (ed.) The use of LSD in psychotherapy and alcoholism. New York, NY: Bobbs-Merrill, 1967, pp.101-116.

46. Hofmann A. LSD, my problem child: reflections on sacred drugs, mysticism, and science. Santa Cruz, 
CA: Multidisciplinary Assoc. for Psychedelic Studies, 2005.

47. Sandison RA. Further studies in the therapeutic value of lysergic acid diethylamide in mental illness. F Ment Sci 1957; 103: 332-343.

48. Sandison R. European symposium on psychotherapy under LSD-25. Göttingen: Göttingen University, 1960.

49. Giberti F and Gregoretti L. [Contribution to the knowledge of hysteria based on the use of psychotomimetics]. Arch Psicol Neurol Psychiatr 1959; 20: 171-191.

50. Ling $\mathrm{T}$ and Buckman J. The use of lysergic acid in individual psychotherapy. Proc R Soc Med 1960; 53: 927-929.

51. Soskin RA. The use of LSD in time-limited psychotherapy. $\mathcal{F}$ Nerv Ment Dis 1973; 157: 410-119.

52. Sessa B. Can psychedelics have a role in psychiatry once again? Br F Psychiatry 2005; 185 : 457-458.

53. Van den Bergh O, Witthöft M, Petersen S, et al. Symptoms and the body: taking the inferential leap. Neurosci Biobehav Rev 2017; 74: 185-203.

54. Perez DL, Dworetzky BA, Dickerson BC, et al. An integrative neurocircuit perspective on psychogenic nonepileptic seizures and functional movement disorders: neural functional unawareness. Clin EEG Neurosci 2015; 46: 4-15.

55. Boeckle M, Liegl G, Jank R, et al. Neural correlates of conversion disorder: overview and meta-analysis of neuroimaging studies on motor conversion disorder. BMC Psychiatry 2016; 16: 195.

56. Brown RJ and Reuber M. Towards an integrative theory of psychogenic non-epileptic seizures (PNES). Clin Psychol Rev 2016; 47: 55-70.

57. Edwards MJ, Adams RA, Brown H, et al. A Bayesian account of "hysteria." Brain 2012; 135 : 3495-3512.

58. Pick S, Goldstein LH, Perez DL, et al. Emotional processing in functional neurological disorder: a review, biopsychosocial model and research agenda. F Neurol Neurosurg Psychiatry 2019; 90: 704-711.

59. Brown RJ, Brunt N, Poliakoff E, et al. Illusory touch and tactile perception in somatoform dissociators. F Psychosom Res 2010; 69: 241-248.
60. Cojan Y, Waber L, Carruzzo A, et al. Motor inhibition in hysterical conversion paralysis. Neuroimage 2009; 47: 1026-1037.

61. Voon V, Brezing C, Gallea C, et al. Emotional stimuli and motor conversion disorder. Brain 2010; 133: 1526-1536.

62. Van Der Kruijs SJM, Bodde NMG, Vaessen MJ, et al. Functional connectivity of dissociation in patients with psychogenic nonepileptic seizures. F Neurol Neurosurg Psychiatry 2012; 83: 239-247.

63. de Lange FP, Roelofs $\mathrm{K}$ and Toni I. Increased self-monitoring during imagined movements in conversion paralysis. Neuropsychologia 2007; 45 : 2051-2058.

64. de Lange FP, Toni I and Roelofs K. Altered connectivity between prefrontal and sensorimotor cortex in conversion paralysis. Neuropsychologia 2010; 48: 1782-1788.

65. de Lange FP, Roelofs $\mathrm{K}$ and Toni I. Motor imagery: a window into the mechanisms and alterations of the motor system. Cortex 2008; 44: 494-506.

66. Buckner RL, Andrews-Hanna JR and Schacter DL. The brain's default network. Ann N Y Acad Sci 2008; 1124: 1-38.

67. Weber ET and Andrade R. Htr2a gene and 5-HT2A receptor expression in the cerebral cortex studied using genetically modified mice. Front Neurosci 2010; 4.

68. Gable RS. Comparison of acute lethal toxicity of commonly abused psychoactive substances. Addiction 2004; 99: 686-696.

69. Nutt DJ, King LA and Phillips LD. Drug harms in the UK: a multicriteria decision analysis. Lancet 2010; 376: 1558-1565.

70. Krebs TS and Johansen PØ. Psychedelics and mental health: a population study. PLoS One 2013; 8: e63972.

71. Grant JE, Lust $\mathrm{K}$ and Chamberlain SR. Hallucinogen use is associated with mental health and addictive problems and impulsivity in university students. Addict Behav Rep 2019; 10.

72. Johnson MW, Richards WA and Griffiths RR. Human hallucinogen research: Guidelines for safety. F Psychopharmacol 2008; 22: 603-620.

73. Hendricks PS, Thorne CB, Clark CB, et al. Classic psychedelic use is associated with reduced psychological distress and suicidality in the United States adult population. F Psychopharmacol 2015; 29: 280-288. 
74. Johansen PO and Krebs TS. Psychedelics not linked to mental health problems or suicidal behavior: a population study. F Psychopharmacol 2015; 29: 270-299.

75. Moreno JL, Holloway T, Albizu L, et al. Metabotropic glutamate $\mathrm{mGlu} 2$ receptor is necessary for the pharmacological and behavioral effects induced by hallucinogenic 5-HT2A receptor agonists. Neurosci Lett 2011; 493: 76-79.
76. Kawa S and Giordano J. A brief historicity of the diagnostic and statistical manual of mental disorders: issues and implications for the future of psychiatric canon and practice. Philos Ethics Humanit Med 2012; 7: 2.

77. Stone J, Smyth R, Carson A, et al. Systematic review of misdiagnosis of conversion symptoms and 'hysteria.' BMF 2005; 331: 989.
Visit SAGE journals online journals.sagepub.com/ home/tpp

(SAGE journals 


\section{University Library}

\section{- M M N E R VA A gateway to Melbourne's research publications}

Minerva Access is the Institutional Repository of The University of Melbourne

\section{Author/s:}

Butler, M;Seynaeve, M;Nicholson, TR;Pick, S;Kanaan, RA;Lees, A;Young, AH;Rucker, J

Title:

Psychedelic treatment of functional neurological disorder: a systematic review

Date:

2020-05-01

Citation:

Butler, M., Seynaeve, M., Nicholson, T. R., Pick, S., Kanaan, R. A., Lees, A., Young, A. H. \& Rucker, J. (2020). Psychedelic treatment of functional neurological disorder: a systematic review. THERAPEUTIC ADVANCES IN PSYCHOPHARMACOLOGY, 10, https:// doi.org/10.1177/2045125320912125.

Persistent Link:

http://hdl.handle.net/11343/244722

License:

CC BY-NC 